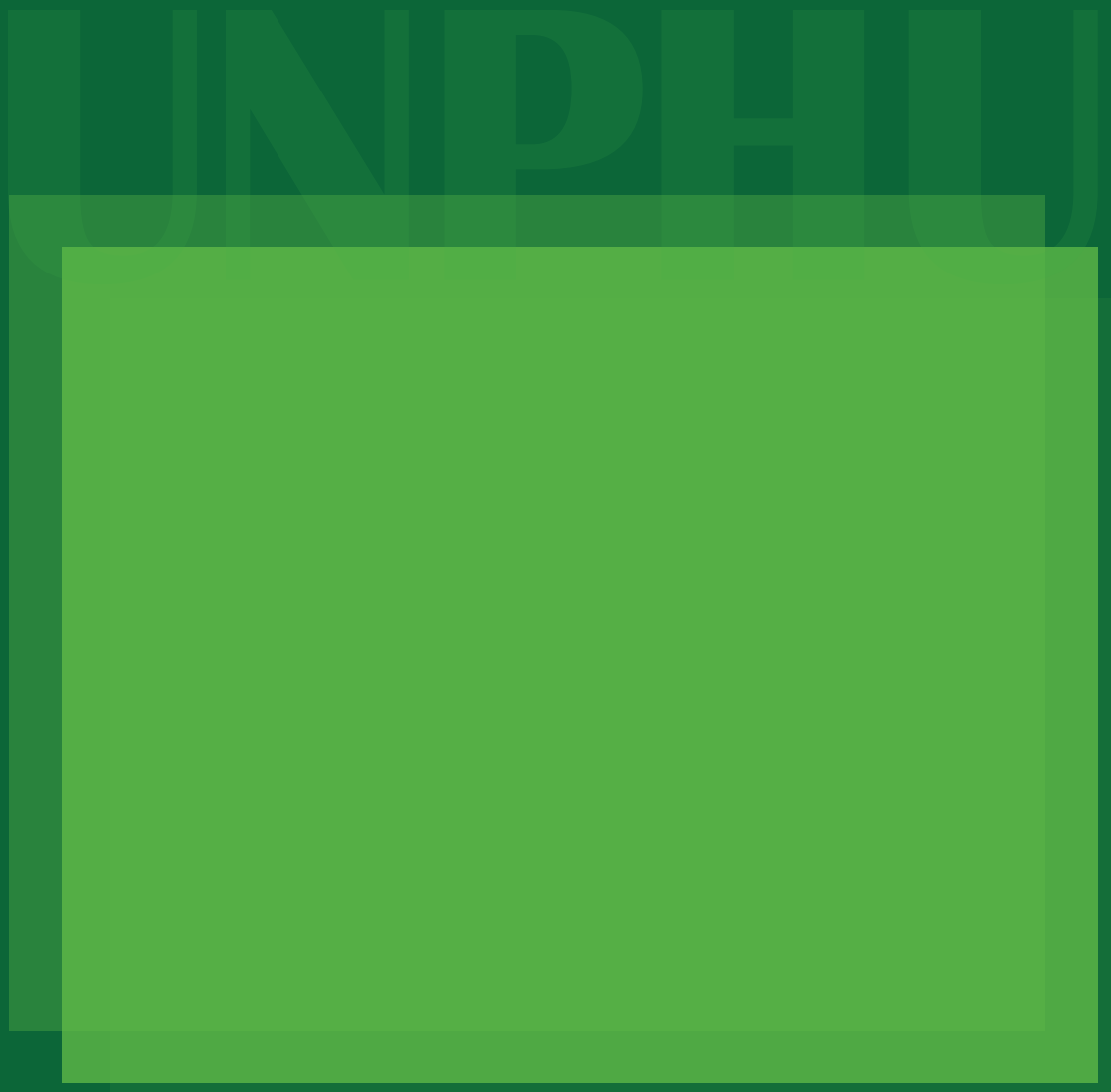




\section{Arquitectura y sostenibilidad en el mundo posmoderno}

\section{Titulos en Ingles}

José Enrique Delmonte Soñé

Arquitecto y escritor. Docente Universidad Iberoamericana

josenriquedelmonte@gmail.com

Fecha de recepción: \# de \#\# de 2017.

Fecha de aceptación: \# de \#\# de 2018.

Favor de citar este artículo de la siguiente forma:

Delmonte, J. E. (2018) Arquitectura y sostenibilidad en el mundo posmoderno

Revista AULA, Vol 62. Número 2, enero junio 2018. Santo Domingo: Amigo del Hogar.

\section{RESUMEN}

El cambio climático exige la redefinición de los patrones de consumo de los seres humanos. Sin embargo, la posmodernidad y sus estilos de vida promueven e inducen a la adquisición de bienes de manera desenfrenada, símbolo del bienestar. Las nuevas geografías urbanas contribuyen a ese ideal posmoderno y la arquitectura es pieza clave para satisfacer las exigencias estéticas de una sociedad consumista. Para el arquitecto, la disyuntiva radica en contribuir con ese esquema predominante o innovar con alternativas de diseño concretas y simples, que reduzcan el impacto de su obra en el medio ambiente.

Palabras clave: posmodernidad, consumo, bienestar, arquitectura, sostenibilidad.

\section{ABSTRACT}

The climate changes demand the redefinition of the patterns of consumption from the human beings. Nevertheless, the postmodern era and its ways of life promote and induce the acquisition of goods in a rampant way. The new urban geographies contribute to this postmodern ideal and the architecture is an essential key in order to satisfy the aesthetic requirements of a consumer society. For the architect, the dilemma comes from contributing with this predominant scheme or from innovating with precise and simple alternatives of design, which reduces the impact of their work in the environment.

Keywords: Postmodern era, consumption, Well-being, architecture, sustainability. 


\section{Arquitectura y sostenibilidad en el mundo posmoderno}

\section{La crisis de la posmodernidad}

[La promoción de un mundo mejor a través de la tecnología, el consumo y el individualismo]

De acuerdo a los últimos informes, la humanidad está en un momento crítico debido al calentamiento global, un fenómeno que altera los procesos que permiten la existencia de vida en el planeta. La información más aceptada es que los hábitos actuales de los seres humanos han producido daños irreversibles a los sistemas y ambientes. La cadena de la vida está, por tanto, amenazada.

Estos hábitos están insertados en la esencia misma de la posmodernidad, la cual que encierra la complejidad de "una sociedad donde impera la insatisfacción como base de la angustia existencial, por mor de la lógica absurda del consumismo" (Mármol, 2016) y cuyos paradigmas envuelven ideales de bienestar basados en el consumo de bienes materiales. De acuerdo a esto, la base sobre la cual el individuo establece su bienestar ya no se sustenta en el "ser" sino en el "poseer" $y$, en consecuencia, la intensa demanda de posesiones materiales provoca una producción extraordinaria de bienes provenientes de recursos limitados y agotables.

La actual crisis económica mundial refleja esta distorsión, donde la necesidad de adquisición de bienes ha superado la capacidad de generación de riqueza real de las naciones (Soros,1999). Sin embargo, la esencia del sistema capitalista exige mayor consumo para poder crecer y superar momentos de instabilidad en su estructura. Por consiguiente, se apuesta por la inducción al consumo y a la generación acelerada de bienes.

La fórmula ha puesto de manifiesto la in- viabilidad de la tecnología como garante del bienestar colectivo y la crisis, por tanto, toca aspectos filosóficos, económicos, sociales e individuales. El incremento del consumismo genera, a su vez, un incremento de la entropía. Provoca agotamiento de recursos y abundancia de desechos $\mathrm{y}$, dada la tendencia de adquisición de bienes - muchas veces por la obsolescencia inducida que contienen los bienes industrializados-, la entropía crece en grandes proporciones. Esta "cultura de obtener y desechar", producto de una economía lineal (Hermida y Domínguez, 2014), se ve sometida a incentivos de la publicidad, del comercio, de las entidades de servicios y de los engranajes que sostienen el estándar de la sociedad. Un artefacto electrónico de más de seis meses de vigencia se convierte en una pieza obsoleta. Se adquiere otro más actualizado y se desecha el anterior. El gasto energético, por tanto, es inmenso y los efectos negativos para la biodiversidad son catastróficos.

Es importante reconocer que la entropía es un proceso ineludible en el desarrollo de los sistemas de producción $\mathrm{y}$, de acuerdo a la teoría de la entropía, todo proceso conduce al caos y a la disolución de su propia condición debido a que se transforma en otra realidad. Se establece que, cuando la energía se transforma, una parte de esa energía se pierde en el proceso, es decir, no está en condiciones de realizar trabajo útil (Rifkin, 2002). Para hacer más entendible este concepto, se puede resumir que la posmodernidad está condenada a su transformación, a su entropía, debido a que agota cada vez más los recursos que le sustentan para ofrecer los bienes materiales a una humanidad en crecimiento. De acuerdo a las teorías de George G. McCurdy (1924), se entiende la experiencia 
humana como un viaje evolutivo dirigido hacia el aprovechamiento de una cantidad cada vez mayor de la energía disponible.

Los actuales hábitos de conducta del ser humano según Navarro (2002), conducen a un callejón sin salida para su propia preservación, y este depende cada vez más de sistemas electrónicos que le facilitan la vida y le transmiten cierta sensación de bienestar. En consecuencia, se apuesta al beneficio que aporta la tecnología para ganar tiempo y, en última instancia, la velocidad se convierte en la pieza clave del bienestar de nuestra época (Koselleck, 2001). Pero, lejos de incrementar el bienestar, la velocidad exige esfuerzos mayores y encierra grandes complejidades para el logro de la satisfacción esperada, bajo un esquema de alto consumo energético. Se produce una diferencia acumulativa entre el consumo energético y la posibilidad de producirlo. Consumir energía emite desechos inservibles que aumentan al desequilibrio ambiental sin que se pueda revertir el proceso negativo. La entropía contribuye, por tanto, a la crisis de la posmodernidad.

"Sobre ti edificaré..."

[Incidencia de la arquitectura en la paradoja posmodernista]

Renzo Piano define a la arquitectura como servicio:

"La arquitectura es un oficio de servicio, pues eso es lo que es: un servicio. La arquitectura es un oficio complejo porque el momento expresivo formal es un momento de síntesis fecundado por todo aquello que se encuentra detrás de la esperas; la geografía y la antropología, el clima, la cultura de cada país donde se va a trabajar; y, de nuevo, la ciencia y el arte" (Piano, 2005).
Como servicio, ofrece soluciones a múltiples demandas que el individuo y la ciudad le establecen, la mayoría de las cuales son reflejos de sus relaciones espacio-temporales que se manifiestan a través de efectos visuales, recorridos, sensaciones y símbolos del presente. Sin embargo, la arquitectura es un instrumento poderoso del mercado de bienes y servicios cuya dinámica se sustenta en la oferta y la demanda. En este juego de equilibrio, la obra construida es un eslabón fundamental entre los materiales -industrializados o simples- y su uso final. Desde que se concibe, la propuesta arquitectónica es un elemento de consumo energético. De hecho, los estudios sobre el impacto que ejerce la arquitectura sobre el medio ambiente indican que los edificios son los mayores consumidores energéticos del planeta y uno de los mayores consumidores de recursos no renovables. A lo largo de su vida útil, un edificio consume materiales de manera permanente y su uso obliga al suministro de energía transformada en iluminación, climatización, agua potable, transporte interno y sistemas de seguridad, además del gasto energético que significa su mantenimiento en el tiempo. (Piano, 2005).

Los daños ecológicos que produce la industria de la construcción son significativos. Un edificio utiliza materiales no renovables en altas cantidades y consume grandes cantidades de agua potable durante su proceso constructivo. La base de la construcción contemporánea es el cemento que, complementado con el acero, constituye un binomio de materiales de alto impacto sobre el medio ambiente. En numerosos estudios realizados en los últimos años, incluso por empresas productoras de cemento, se ha determinado que las fábricas de cemento emiten "grandes masas de partículas de polvo con alto contenido de calcio, magnesio, dióxido de carbono entre otros, son emanadas al espacio, contaminando el aire, aumentando el $\mathrm{pH}$ 
del suelo retardando el crecimiento de la vegetación por un lado y por el otro, la descarga de aguas residuales de origen industrial producto del mismo proceso" (Ávila, 2015) Muchos materiales de terminación son producidos con elementos altamente tóxicos y en su fabricación se invierten grandes cantidades de energía o de agua, y producen, además, desechos que no pueden convertirse en algo útil. De igual modo, estos materiales atraviesan grandes distancias desde su origen hasta la fábrica y desde la fábrica hasta el lugar donde se realiza la obra de construcción, situación que se adiciona al gasto de combustibles fósiles y la emisión de $\mathrm{CO} 2$ a la atmósfera, necesarios para su transportación. La importación de materiales desde el extranjero incrementa, aún más, el impacto negativo al medio ambiente.

Estas condiciones básicas deben complementarse con los efectos secundarios que provoca un edificio durante su vida útil, en cuya lista se incluye las enfermedades laborales o los accidentes y riesgos que provocan a sus usuarios. Inadecuada iluminación natural o climatización, abundancia de partículas de polvo, ruidos, olores, o mala distribución, conducen al incremento de daños físicos y psicológicos en los usuarios los cuales, a su vez, aumentan el consumo de servicios de salud y el deterioro de la calidad de vida tanto del usuario como de sus familiares. Desde 1982, la Organización Mundial de la Salud ha incluido en sus informes el Síndrome del edificio enfermo (SEE), definido como el "conjunto de síntomas diversos que presentan, predominantemente, los individuos en estos edificios y que no van en general acompañados de ninguna lesión orgánica o signo físico, diagnosticándose, a menudo, por exclusión" (Ávila, 2005).

Todas estos fenómenos de impacto ambiental que provocan los edificios se incrementan en la medida en que aumenta la huella urbana, es decir, el incremento del tamaño de las ciudades en el territorio. La posmodernidad promueve la figura del individuo vinculado a los patrones urbanos como símbolo de éxito, apegado a la tecnología y al consumo de productos importados exhibidos en los grandes centros comerciales. Koolhass (2004) plantea la interdependencia de los medios de producción con las expectativas de bienestar que el presente promueve, los centros urbanos se convierten en la aspiración existencial del hombre actual, "cuya confirmación de sentirse parte del sistema radica, en esencia, en el fenómeno participar del espectáculo urbano" (Amendola,2000,pp.211-228). Por tanto, el crecimiento poblacional se produce en las ciudades de forma acelerada, en contraposición con el abandono de las zonas rurales.

Sin embargo, la ciudad sigue siendo el factor más importante en el daño ecológico del planeta debido a todas las variables que intervienen en el sistema de convivencia actual. De acuerdo a la Agencia de las Naciones Unidas para los Asentamientos Humanos -ONU/ Hábitat (2015), el problema ambiental de las ciudades es un fenómeno en crecimiento y de alta preocupación para el equilibrio ecológico, por su incidencia en el calentamiento global. El panorama es alarmante, ya que las ciudades ocupan solo el $2 \%$ del territorio global, consumen el $60 \%$ de la energía, emiten el $70 \%$ de gases de invernadero y producen el $70 \%$ de los desechos. Cada edificio en particular produce grandes cantidades de desechos y contribuye al calentamiento global a través de sus cubiertas y sus superficies adyacentes. En la medida en que se incrementa la densidad de edificios se eleva el efecto de calor en su entorno, medido a través de las llamadas islas de calor presentes en el escenario urbano alrededor del planeta. 
Richard (1995), define una isla de calor urbana como:

"El calor característico tanto de la atmósfera como de las superficies en las ciudades (o áreas urbanas) comparadas con sus entornos no urbanizados. La isla de calor es un ejemplo de modificación climática no intencional cuando la urbanización le cambia las características a la superficie y a la atmósfera de la tierra". (pp.81-107)

Como consecuencia, se produce una modificación significativa en las condiciones ambientales urbanas tales como:

"Aumento de temperatura, migración de la fauna de los parques, baja captura de precipitación pluvial, impacto a suelos, aumento del uso de energía eléctrica por los aires acondicionados, mayor incidencia de problemas respiratorios por la contaminación, incremento del ruido, menor captura de dióxido de carbono (uno de los principales gases que genera la inversión térmica) y falta de producción de oxígeno".

(Carrillo, 2005:8)

La enorme concentración humana en las ciudades desborda la capacidad de los sistemas urbanos y atenta con el bienestar colectivo, más allá de la imagen de modernidad que proyecta (Mayr,2009). Detrás de esa máscara radican contradicciones profundas. El gasto energético que supone mantener en funcionamiento todo el sistema urbano, tales como movilidad, seguridad, habitabilidad, ocio, comercialización, institucionalidad, controles, servicios públicos, salud, educación, ornato, entre otros, es enorme y encierra el fenómeno entrópico en su estructura. Cada día es necesario invertir mayores recursos para que el aparato urbano funcione, cuyas consecuencias se vislumbran en la concentración de desechos, la polución, los daños en la salud física, ambiental y emocional de sus habitantes, así como en el caos, la inseguridad y la inequidad que la entropía urbana produce (Fariña, 2002).

\section{Muchos mañanas posibles y una sola elec- ción}

[La impostergable tarea de repensar la arquitectura del presente]

Los movimientos ecologistas han asumido el reto de exigir y promover la reducción del impacto negativo que los territorios urbanos provocan, a través de numerosas propuestas dirigidas a motivar cambios de conducta individual y colectiva. Varios estudios indican que la eliminación de prácticas aprendidas en la concepción del diseño arquitectónico y en la industria de la construcción, es un paso importante para reducir la enorme huella ecológica que la arquitectura produce y que la ciudad multiplica (Hernández, 2010).

Existen numerosos conceptos y denominaciones que forman parte del lenguaje conservacionista de los movimientos ecológicos, que se resumen, básicamente, en la arquitectura sostenible. Larraga (2014) incluye nombres como el de arquitectura solar, bio-arquitectura, arquitectura verde, bioclimática hasta las eco villas; pasando desde los movimientos tecno-centristas hasta aquellos eco-centristas; dando un panorama general del estado del arte de la sustentabilidad en la arquitectura, así como las distintas percepciones que enriquecen y aportan a la complejidad y al debate de la sustentabilidad.

Sin embargo, las complejidades e intereses en el mercado de bienes raíces -al que la arquitectura está vinculada- y en el escenario político y empresarial, dificultan la incorporación de nuevas prácticas dirigidas a soluciones menos agresivas. De hecho, la visión de producir una 
arquitectura más responsable desde el punto de vista ambiental, se ha convertido en un territorio minado para los diseñadores, quienes se enfrentan a grupos con diferentes objetivos y con soluciones que encierran posiciones extremas. Lárraga (2014) ha propuesto un mapa de aproximación a la arquitectura sostenible que incluye, desde las posturas de alta tecnología apoyadas en el marketing, hasta aquellas llamadas "transformacionistas", que incluyen el ecofeminismo, ecosocialismo, anticapitalista y de posturas donde predominan conceptos como la equidad, autonomía, gobernanza, autosuficiencia, con propuestas integradoras, incluyentes, democráticas, que se niegan al status quo oponiéndose y resolviendo el hábitat con principios diferentes a los convencionales, siendo el caso de las ecovillas y ecoaldeas.

Poco a poco, el tema ha ganado espacio en las escuelas de arquitectura de todo el orbe. Sin embargo, de acuerdo a la posición que ellas adoptan, los futuros arquitectos son entrenados bajo criterios que de acuerdo con Chesbrough y Crowther (2006), se vinculan a un extremo $\mathrm{u}$ otro, es decir, a las tendencias High Tech, -con proyectos influenciados por el Star System o arquitectos estrellas- o las menos sofisticadas o primitivas apegadas al extremismo conservacionista (Rivero, 2012). Ambos casos pueden reforzar la conciencia ecológica en el diseñador, pero con enfoques diferentes. Es obvio que en las escuelas pertenecientes a países con mayor desarrollo tecnológico se induce a soluciones tecnificadas y al uso de métodos sistematizados que basan su eficiencia en equipamientos industrializados. Sin embargo, con mayor frecuencia surgen tendencias académicas que reincorporan prácticas tradicionales de soluciones simples y de bajo impacto ambiental. Pierri (2005) las divide en "sustentabilidad fuerte y sustentabilidad débil" de acuerdo a la posición que se escoja para promover la buena práctica de arquitectura sostenible, y propone la "posición reformista" que abarca, tanto elementos de la sustentabilidad fuerte, como de la débil, de acuerdo a las posibilidades que el contexto permite.

El sistema económico actual ha reconocido el poder atrayente de la arquitectura y los centros financieros mundiales se esfuerzan por poseer "edificios espectáculo", capaces de atraer inversionistas y convertirse en territorios de alta competitividad (Vargas,2009). Es un reflejo de la "mundialización de la arquitectura" que sirve de estandarte a los nuevos procesos de producción de la globalización que, de acuerdo a Saskia Sassen (1999), genera nuevas geografías urbanas en el territorio. Zaida Muxí (2009) señala que "esta situación ha conducido a pugnas entre las ciudades para conseguir ocupar un lugar en las nuevas redes de producción y, en consecuencia, la ciudad se presenta como si de un individuo aislado se tratara, contradiciendo, por tanto, la idea de red como estructura de funcionamiento" (Sassen, 1999).

Esta lucha forma parte del ideal posmoderno que envuelve a la cultura y en particular a la arquitectura. Como elemento fundamental de validación de la imagen de actualidad que las ciudades desean poseer para poder atraer grandes capitales del mundo corporativo, los edificios deben reflejar los códigos estéticos que la tecnología promueve a través de formas y sistemas propios del nuevo engranaje económico-cultural. Así las cosas, la arquitectura sucumbe ante las exigencias de un consumidor que busca efectos inmediatos a través de la imagen y de sus componentes físico-espaciales.

Sin embargo, Sassen (1999) entiende que estas soluciones altamente tecnificadas contrastan con el contexto donde se insertan, ya que producen impactos en el territorio que desarticulan ambientes tradicionales y sobrevaloran el suelo. De esta forma, la dinámica urbana pro- 
voca tensiones que según Hernández y Delgado (2010) dan como resultado efectos inesperados, tales como desarticulación, desvinculación, conurbación y la gentrificación (Díaz, 2013), los cuales fueron denominados por Rueda (1997) como impulsadores de cambios traumáticos en la estructura urbana tradicional.

Todas estas derivaciones quedan subyugadas al marketing y a la divulgación de ideales de bienestar, modernidad y desarrollo que, lejos de alcanzar a la mayor cantidad de habitantes de la ciudad, termina elevando los indicadores de segregación, discriminación e inequidad. De hecho, ONU/Hábitat (2015) ha desarrollado un sistema de evaluación de la prosperidad en las ciudades, basado en la visión de conducir el mundo hacia un futuro urbano próspero en términos económicos, sociales, políticos y ambientales, con el objetivo de medir el impacto de los nuevos cambios en el territorio urbano y plantear soluciones ante sus consecuencias.

En esta carrera de globalización, la ciudad recibe un impacto ambiental negativo debido a los efectos de la movilización de sus usuarios -la conurbación incrementa estos efectos-, la densificación del territorio, la velocidad de cambios -los cuales muchas veces impiden que las ciudades realicen la adecuación de los servicios-, la emisión de gases invernaderos, los ruidos, los gases tóxicos y contaminantes, entre otros, además, de los problemas sociales. En adición, las vías de aplicación de sistemas de reducción de impacto ambiental que los arquitectos deben incorporar en sus proyectos, se dirigen a las soluciones que la sustentabilidad blanda, a través de sistemas sofisticados, promueve.

Alternativas menos industrializadas ligadas a la sustentabilidad dura no son utilizadas con frecuencia, debido, quizás, a su lejanía con los códigos estéticos que la posmodernidad válida para las ciudades. Este abismo entre las dos alternativas impacta directamente en el costo de los edificios, ya que las exigencias para reducir el impacto ambiental inducen a la selección de equipos y sistemas tecnológicos de última generación, cuyos precios son elevados. De hecho, se calcula que la aplicación en países subdesarrollados del Código Leed (Green Building Council,1993), el más representativo en los Estados Unidos en materia de diseño de edificios verdes, incrementa en cerca de un 35\% el presupuesto de construcción de un inmueble.

Algunas voces en el escenario mundial de la arquitectura se hacen escuchar a través de propuestas alternativas de bajo impacto y con costos reducidos, apegados a la valoración de la cultura del sitio y de los patrones sociales de los habitantes, además de promover la sustentabilidad, el sentido de pertenencia y la trasmisión de sentimientos de orgullo e identidad. Sin perder calidad espacial ni ambiental, reflejan un alto compromiso estético y social. El trabajo de Francis Keré (Burkina Faso), Sharon Davis (New York), Shigeru Ban (Japón), Bruno Stagno (Costa Rica) y Oscar Imbert (República Dominicana), por solo citar cinco arquitectos del presente, sirven de ejemplo dentro de un amplio escenario de propuestas sensibles con el medio ambiente alrededor del mundo.

Tanto Francis Keré como Sharon Davis utilizan la tradición constructiva del lugar y la acción comunitaria en su construcción, reduciendo costos significativos y reivindicando sistemas tradicionales a través de un exquisito manejo estético y espacial. Ban, por su lado, el premio Pritzker 2014, ha asumido el compromiso de actuar en zonas de alta vulnerabilidad y pobreza con materiales de desecho y con la incorporación de mano de obra comunitaria para lograr alternativas de refugio viables. Stagno se ha dedicado a introducir soluciones aprendidas de la tradición constructiva del Caribe en proyectos importantes, gracias a una sensibilidad 
ante el medio y la responsabilidad por conservarlo. Oscar Imbert, por último, es un ejemplo de cómo la arquitectura académica logra aprender de la tradición y utiliza lenguajes de la arquitectura popular y vernácula en proyectos de gran escala que afianzan el sentido de identidad del lugar. En común, ellos reflejan una buena práctica arquitectónica con recursos técnicos y estéticos de bajo costo y de bajo impacto en el medio ambiente.

El tema del medio ambiente en los círculos académicos y profesionales ligados a la arquitectura y al urbanismo adquiere, cada día, mayor presencia, debido a la responsabilidad que tienen los diseñadores ante el problema climático. El último encuentro mundial sobre el clima, Paris Climat 2015, ha reafirmado el compromiso de reducción de gases de invernadero y reducción de la polución ambiental en un tiempo corto, ya que los datos que sustentan el deterioro del planeta son irrefutables. El tiempo de lo inagotable ya no existe, el entorno natural se transforma cada día y nuestra capacidad económica está sometida a grandes laberintos de los que aún se desconoce su desenlace, a pesar del extraordinario bienestar que las ciudades exponen.

Se está ante la disyuntiva de aferrarse a los hábitos que han provocado el desequilibrio existente y conducido a un futuro distópico, inhumano e insostenible, o despojarse de ellos y enfrentar los retos con la visión puesta en una utopía posible y permanente. Los arquitectos del presente tienen el futuro en sus manos.

\section{Referencia}

Ávila, J. (2009). Contaminación atmosférica en las empresas cementeras en el marco de la responsabilidad social ante comunidades adyacentes. CICAG, vol. 6, edición 1.
Amendola, G. (2000). La ciudad postmoderna. Madrid, España: Celeste Ediciones.

Carrillo, L. (2005, mayo 2). El efecto isla de calor. Gaceta Universitaria, (número 388), p.8.

Chesbrough,H. y Crowther,A.K. (2006). Beyon High Tech: Early Adopters of Open Innovation in Other Industries. [Más allá de la alta tecnología: Pioneros de la innovación abierta en otras industrias]. R\&D Management. Vol.36,No.3,pp.229-236.

Díaz Parra,I. (2013, junio 25). La gentrificación en la cambiante estructura socioespacial de la ciudad. Revista Bibliográfica de Geografía y Ciencias Sociales, XVIII, No.1030. Recuperado de http://www. ub.es/geocrit/b3w-1030.htm.

Fariña, J., Ruiz, J. (2011, mayo). Orden, desorden y entropía en la construcción de la ciudad". Urban (S.I), volumen 7, p. 8-15.

Green Building Council. (1993). Leadership in Energy \& Environmental Design. Washington D.C, Estados Unidos: Green Building Council. Recuperado de https:// new.usgbc.org/leed

Hermida,C., Domínguez, M. (2014). Economía circular como para el ecodiseño: el modelo ECO-3. Informador Técnico, volumen 78, No.1, pp. 82-90.

Hernández,S., Delgado, D. (2010) enero-junio. Manejo sustentable del sitio en proyectos de arquitectura; criterios y estrategias. Quivera, Volumen 12, No.1), pp. 38-51. 
Koolhass, R. (2004). Delirio de Nueva York. Barcelona, España: Editorial Gustavo Gili S.L.

Koselleck, R. (2001). Estratos del tiempo: estudios sobre la historia. España, Madrid: Paidós. Lárraga, R. (2014). Percepciones de la sostenibilidad en arquitectura: un mapa de las principales propuestas de aproximación. Revista Caribeña de Ciencias Sociales. Recuperado de http://caribeña. eumed.net/sostenibilidad-arquitectura.

MacCurdy, G.G. ( 1924). Humans Origins: A Manual of Prehistory. [ Orígenes de los humanos: Un manual de la prehistoria] D. Apleton and Co., New York .

Maldonado, J. (2009) noviembre. Ciudades y contaminación ambiental. Revista de Ingeniería, volumen 30, p. 66-71

Mármol, J. (6 de enero de 2016). Posmodernidad e identidad líquida. El Nacional, Santo Domingo. Recuperado de http://eldia.com. do/posmodernidad-e-identidad-liquida/

Muxí, Z. (2009). La arquitectura de la ciudad global. Buenos Aires, Argentina: Nobuko.

Navarro, V. (2002). Neoliberalismo y estado del bienestar. Madrid, España: Ariel.

NTP 289: Síndrome del edificio enfermo: factores de riesgo. Instituto Nacional de Seguridad e Higiene en el Trabajo. Ministerio de Trabajo y Asuntos Sociales http://www.insht.es/InshtWeb/Contenidos/Documentacion/FichasTecnicas/ NTP/Ficheros/201a300/ntp_289.pdf
Organización de las Naciones Unidas. (2017). El contexto global según la nueva Agenda Urbana. Recuperado de https://www. habitat3.org/the-new-urban-agenda.

Piano, R. (2005). La responsabilidad del arquitecto. Conversaciones con Renzo Cassigoli. Barcelona, España: Editorial Gustavo Gili, S.L.

Pierri, N. (2005). Historia del concepto de desarrollo sustentable. AA. VV. ¿Sustentabilidad? Desacuerdos sobre el desarrollo sustentable. Zacatecas, México: Universidad Autónoma de Zacatecas.

Oke, T.R. (1995). The head island characteristics of the urban boundary layer: Characteristics, causes and effects. Wind climate in cities. [Características del calor del límite de la capa urbana en la isla : Clima del viento en ciudades]. Kluwer Academic, Netherlands, pp.81-107.

Rifkin, J. (2002). La economía del hidrógeno. Barcelona, España: Editorial Paidós.

Rivero, L. (marzo de 2012). Mujer y Star System arquitectónico. Algunas cuestiones de género en la arquitectura de Zaha Hadid. En A. Suárez (Director), I Congreso Internacional de Comunicación y Género. Congreso llevado a cabo en la Universidad de Sevilla, España.

Rueda, S. (1997). La ciudad compacta y diversa frente a la conurbación difusa. Ciudades para un futuro sostenible. Recuperado de http://habitat.aq.upm.es/cs/p2/ a009.html. 
Sassen, S. (1999) La ciudad global: Nueva York, Londres, Tokio. Buenos Aires, Argentina: Eudeba.

Soros, G. (1999). La crisis del capitalismo global. La sociedad abierta en peligro. Buenos Aires, Argentina: Editorial Sudamericana.
Vargas, M. (3 de mayo de 2009). La arquitectura como espectáculo. El país. Recuperado de http://elpais.com/diario/2009/05/03/ opinion/1241301610_850215.html.

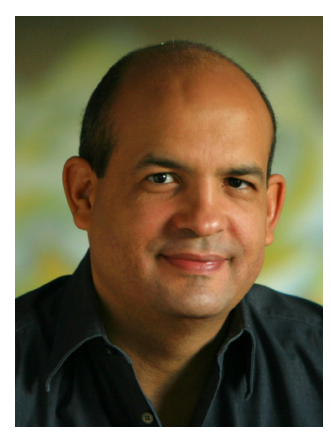

\section{José Enrique Delmonte Soñé}

Arquitecto egresado de la Universidad Nacional Pedro Henríquez Ureña, posgrados en arquitectura, urbanismo e historia en University of Florida y en la Universidad Nacional de Tucumán. Especialista en Arquitectura Sostenible por la University of Georgia, Máster en Conservación de Monumentos y Bienes Culturales por UNPHU y Doctorado en Lingüística y Literatura por la Pontificia Universidad Católica Madre y Maestra. Es un reconocido historiador e investigador de la arquitectura y el urbanismo dominicanos. Ha realizado una larga labor docente y ha sido profesor invitado en universidades extranjeras. Ocupó la posición de decano de la Facultad de Arquitectura y Artes de la UNPHU y viceministro de Patrimonio Cultural. Ejerce como arquitecto bajo su firma privada y en la actualidad es docente en la Escuela de Arquitectura de la Universidad Iberoamericana (UNIBE). 